\title{
6. What shapes the development of Indigenous children?
}

\author{
Carrington Shepherd and Stephen R. Zubrick
}

Descriptions of the Australian Indigenous circumstance have been dramatically enriched through improvements in, and delivery of, high quality quantitative survey findings over the past 20 years. Since 1901 - when Indigenous Australians were effectively excluded from even being counted in the populations of the States of the Commonwealth (Briscoe 2003) - Australia has made significant improvements in its capacity to detail the demographic and developmental status of its Indigenous peoples. Amid this progress though, it still remains the case that good quality descriptions of the developmental circumstances of Indigenous children, as distinct from Indigenous adults, are surprisingly few and far between. The 2008 National Aboriginal and Torres Strait Islander Social Survey (NATSISS) provides an opportunity to specifically describe the health and development of Indigenous children using an important and high quality data source.

To make sense of the findings about the health and development of Australian Indigenous children, it is important to place their development in the context of the healthy development of all children. This is not to discount the vital and obvious importance of Indigenous culture. Rather, by starting with some principles of healthy development that apply universally to all children, some of the underpinnings of the current Australian Indigenous circumstance and its apparent intractability are brought into perspective. We believe this opening perspective offers opportunities for better policies, services and practices to improve the life prospects of Australian Indigenous people.

\section{Early development in the course of human development}

Healthy development in early life is important for all children. The empirical literature now provides abundant evidence confirming that a child's developmental pathway shapes the subsequent course of their life. Child development is influenced not just by what occurs in infancy and beyond, but by processes that take place in-utero and prior to conception. Exposures in the 
earliest stages of life - such as the effects of maternal drug use, for example can affect early brain development and play a critical role in shaping health prospects into adulthood (Keating and Hertzman 1999).

The evidence-base supporting healthy child development has been built-up over the course of decades, from research fields as diverse as neurobiology, psychology and social sciences, and confirms that children and their development have many spheres of influence - as depicted in Fig. 6.1. A child's immediate family and the household environment have the most direct impact on their development, although extended family networks, schools, formal services, neighbourhood characteristics and elements of the broader social, economic and political society, can all impact on a family's ability to provide the necessary support to a child's development (Bronfenbrenner 1979; Jessor 1993).

\section{Fig. 6.1 Children within contexts of influence}

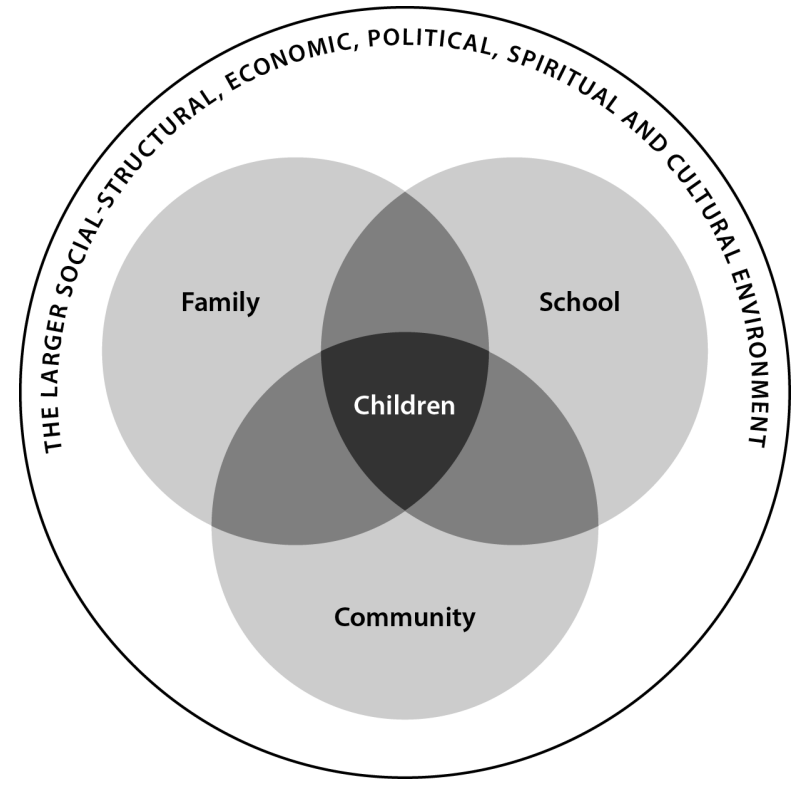

Source: Jessor 1993

The experiences of children at home and in daycare from birth to age of entry into kindergarten play a substantial role in their development, particularly in early cognitive and language development and in emotional and behavioural regulation. Young children who are well nurtured do better in school and develop the skills needed to take their place as productive and responsible adults (Zubrick et al. 2006).

Understanding the relationships between the factors that influence child development and their timing is important if communities and governments 
are to take appropriate action to ensure a fair start for all children. Nurturing children in their early years is vital for attacking the worst effects of disadvantage. Governments around the world are now seeking better ways to re-invest in their human service infrastructure to better meet the needs of children in order to bring about population-level improvements in health and human capability. The emerging consensus is that the greatest gains in overcoming disadvantage are likely to be achieved through universal preventions which give all children a better start in life. This is the preferred policy approach to reducing poverty being advocated by international agencies such as UNICEF and the World Bank and has been termed 'human development though early child development' (Young 2002).

Human development is broadly about expanding human capabilities, so that individuals can participate economically, socially and civically and choose lives that they value. Childhood is centrally located in models of human development, as it represents a critical period where skills are acquired and accumulated for benefit throughout the lifecourse. In the progression from childhood onwards there is a general consensus of evidence that human capability is optimised when individuals:

- are able to regulate their emotions

- are able to engage in exploratory behaviour

- are able to communicate effectively

- are self-directed

- have intellectual flexibility

- possess some degree of introspection, and

- possess self-efficacy in meeting life's challenges.

How these seven 'strengths' develop in childhood are critical in enabling onward capability - in essence, those that start at a low 'threshold' are likely to lose opportunities for further development at later stages in life (Zubrick 2010).

Quite importantly, the evidence in the child development literature supports a relatively small set of mechanisms that change developmental strengths. These mechanisms work in one of three ways - they either prompt, facilitate or constrain the development and maintenance of strengths. While these mechanisms will be elaborated on later in the paper, it should be noted that they operate similarly among Indigenous and non-Indigenous children, albeit in vastly different population contexts. They also operate across the lifecourse. Fig. 6.2 offers a lifecourse perspective on child development in the context of a selection of global and national events from 1945 to present, and highlights the variation in a hypothetical outcome of interest from birth to late life. The outcome could take many forms including specific health conditions, general 
health status, mental health, and any of the developmental strengths cited above. The variation in the outcome of interest can be thought of as the variation in the lifecourse of an individual life or as a time series of the population estimate over the relevant period. The occurrence of parental divorce, the onset of smoking and alcohol abuse, the sudden closure of an industry and unemployment are included as examples of exposures of interest. All of these exposures (and the outcome) can be influenced by broader, macrosocial factors - these include global and national events that occur over time, such as the introduction of free higher education in the 1970s or the emergence of the World Wide Web in the 1990s (Zubrick et al. 2009).

This paper uses a human development framework to explore the developmental status of Indigenous children in Australia, using data from the 2008 NATSISS. We examine how Indigenous children are faring in terms of some traditional markers of child development and the mechanisms that prompt, facilitate and constrain Indigenous child development. We also explore what the NATSISS can tell us about the relative importance of factors that influence key child development outcomes.

\section{Child development and the 2008 NATSISS}

The NATSISS is a vital source of data for addressing the human capability story in an Australian Indigenous context. The 2008 NATSISS is significant in that it enables, for the first time, an examination of the development of children. As such, it is one of the few reliable quantitative resources that have detailed information on both developmental outcomes and their risk factors for Indigenous persons aged $0-14$ years.

In terms of child developmental outcomes, the 2008 NATSISS asked about birthweight and gestational age (for 0-3 year olds only), a global question on health status, and questions regarding specific problems with ears/hearing, eyes/sight and teeth/gums. In addition, some information can be gleaned on educational attendance. All responses for 0-14 year olds were provided by parents/guardians in most instances, or a member of the household with responsibility for the child. In addition, the NATSISS included a rich set of variables that can be described as either prompts, facilitators or constraints of child development. These include aspects of diet and nutrition, connection with culture, carer education, informal learning, stress and supports. 


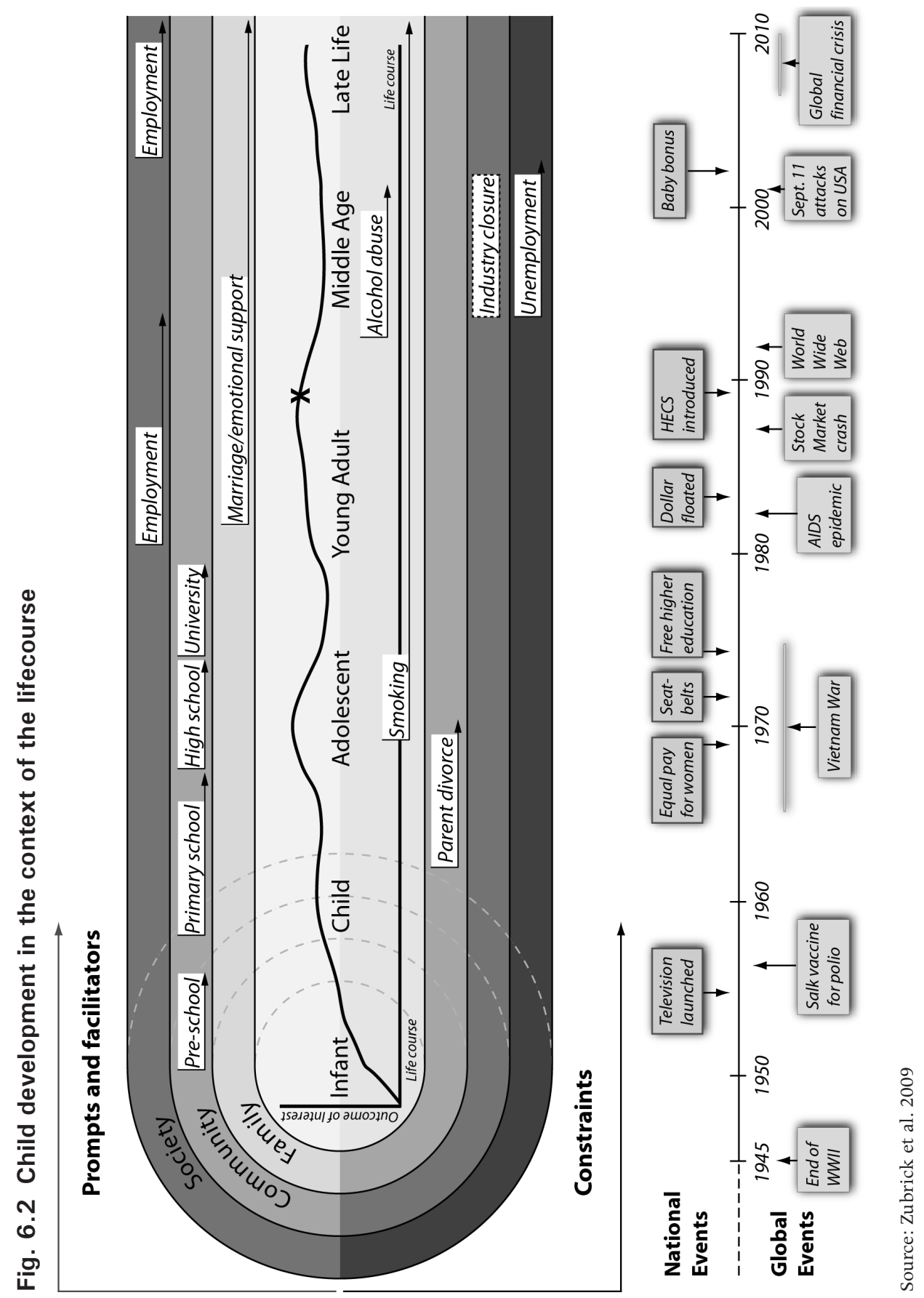


The large scale of the 2008 NATSISS (almost 5500 children were sampled) enables a robust analysis of child developmental outcomes and their antecedents, with potential for regional comparisons (by State/Territory or geographic remoteness). All analyses in this paper were conducted on the State/Territory by ASGC Remoteness Structure Confidentialised Unit Record File (CURF), accessed via the Australian Bureau of Statistics (ABS) Remote Access Data Laboratory (RADL). This CURF provided a dichotomous national remoteness data item (remote/ non-remote) and a 13-part derived item that cross-classifies State/Territory by remoteness.

The depth (in terms of sample size) and breadth (in terms of data items) of the 2008 NATSISS offer considerable strength for the purposes of examining aspects of Indigenous child development. However, there are, as with any study, a range of limitations to the NATSISS data which restrict what can be achieved in this paper. First, the NATSISS uses a cross-sectional design which reduces any discussion of causal inference to a discussion about associations. Second, there is a lack of information on academic performance and social and emotional wellbeing, and a narrow range of educational attendance variables in the survey. This limits our ability to examine some of the key domains of child development. Third, all of the questions relating to $0-14$ year olds rely on the perceptions and recall of parents and caregivers, which are inherently open to issues of bias (e.g. problems with interpretation, willingness to answer openly) and inaccuracy (Sen 2002). In relation to the 2008 NATSISS, the difficulties with interpreting a child's health are two-fold - a carer's views may not accord with that of a medical expert or with the view of the child themselves. Fourth, the available CURFs do not allow a full examination of the effects of geographic location. This is particularly limiting in Indigenous contexts because of the heterogeneity of Australian Indigenous population groups (Bell 1995) and the relative importance that a sense of place and connection to land has on the health of Indigenous peoples (Boddington and Raisanen 2009; Saggers and Gray 2007). Lastly, the CURF does not include stratum or Collection District (CD) information which precludes a multi-level analysis of the data, and therefore we are not able to fully examine the relationships between factors at the individual, family and neighbourhood level and child health outcomes.

\section{Developmental outcomes for Indigenous children}

Before we begin to describe the developmental status of Indigenous children we must ask the question, 'what constitutes an outcome?' In this paper, the overarching outcome is the capability to participate - economically, socially and civically. These outcomes are largely at the core of what public policy and its funding effort seeks to achieve. Public policy and expenditure on human 
services is deliberately organised to influence human capability with the express aim of enabling more people to choose lives that they value. There has been a heavy emphasis historically on economic participation and only in recent times have developed countries begun to listen to citizen demands that there is more to life than participating in the labour market - social participation and civic participation form part of the mix of what human development is all about (Fukuda-Parr and Kumar 2004).

Focusing on a human capability framework enables us to examine specific types of outcomes, i.e. diseases, good health, literacy, as well as those that may be considered as developmental 'means'. For example, the achievement of good health or the occurrence of specific diseases, are typically studied as 'outcomes' in their own right. These outcomes may also be thought of as the means through which the capability to participate economically, socially and civically is achieved or diminished. Using this as a guiding framework, we have selected seven outcomes for children from the 2008 NATSISS:

- birthweight

- gestational age

- overall (global) health status

- eye/sight problems

- ear/hearing problems

- teeth and gum problems, and

- educational attendance.

\section{Birthweight}

High rates of low birthweight in developing countries are primarily due to intrauterine growth restriction, which is associated with a range of poor outcomes that commence at birth (death, disability and poor health) and can lead to complications in childhood and the development of chronic illnesses in adult life (Australian Institute of Health and Welfare et al. 1999; Ford et al. 2003; Zubrick et al. 2008). Low birthweight babies are generally more prevalent in Indigenous populations, where population rates correspond more closely with those observed in developing nations (Steering Committee for the Review of Government Service Provision (SCRGSP) 2009).

Low birthweight is typically defined as less than 2500 grams, while those born less than 1500 grams are of very low birthweight. The NATSISS found that 11.2 per cent of Indigenous children aged 0-3 years in Australia were of low birthweight and 1.9 per cent were born at very low birthweight (Table 6.1). 


\section{Gestational age}

Babies born prior to 37 weeks gestation are considered to be 'preterm' or to have 'low' gestational age. This cut-off point aligns with the development of several organ systems, and evidence suggests that low gestation is associated with a greater risk of neonatal mortality and a range of morbidities into childhood and beyond (Kuh and Ben-Shlomo 2004). Close to one-quarter (24.1\%) of Indigenous children aged 0-3 years were considered preterm at birth. A higher proportion of females than males were preterm $(26.9 \%$ compared with $21.4 \%)$.

\section{Global health}

Global health status was assessed on a five-point ordinal scale: excellent; very good; good; fair; or poor. Less than 4 per cent of children aged 0-14 years had fair or poor health. The majority were in either excellent $(46 \%)$ or very good health $(32 \%)$. There was some variation by age, with older children generally less likely to be in excellent or very good health than younger age groups. This pattern can be observed in both non-remote and remote areas (see Fig. 6.3).

\section{Fig. 6.3 Proportion of Indigenous children in excellent or very good} health, by age, Australia, 2008

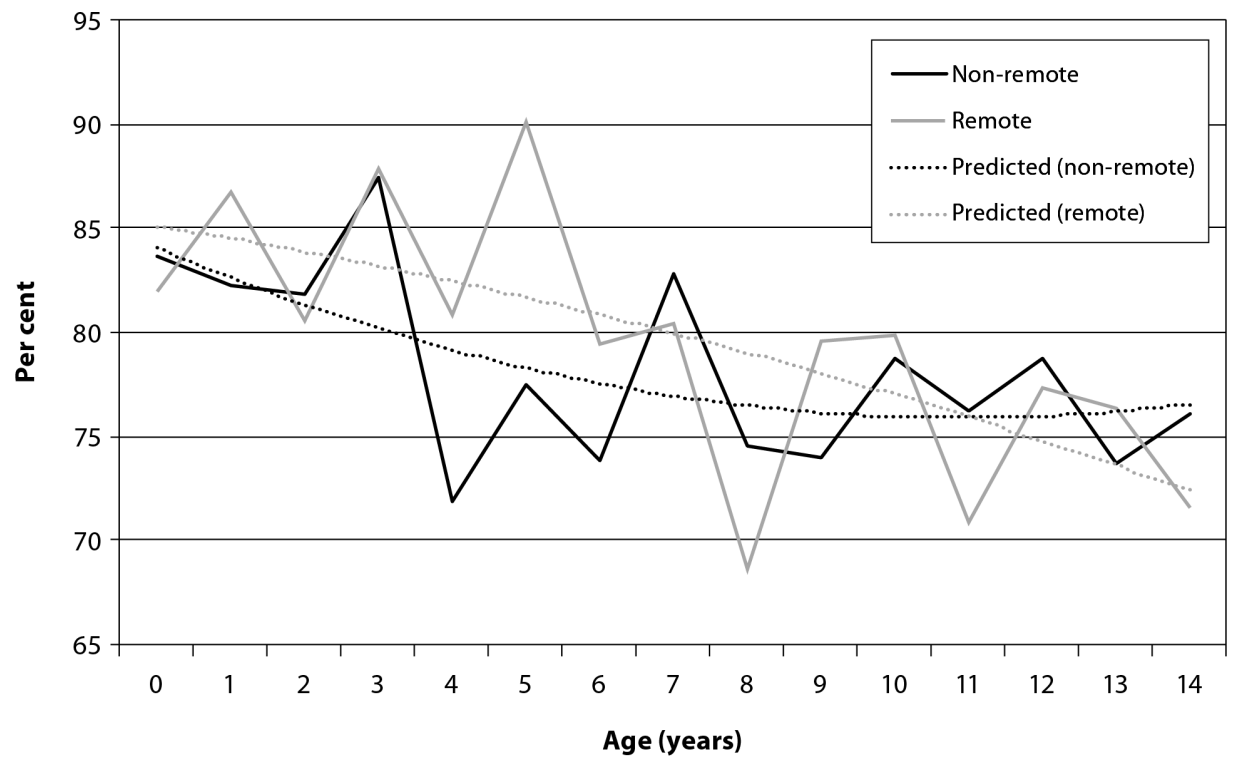

a. The broken lines represent a 'best fit' line, based on the coefficients of a second order polynomial regression.

Source: Authors' customised calculations using the 2008 NATSISS (accessed using the RADL) 
When aggregated together, global health levels were broadly similar in remote and non-remote areas of Australia - however, this masks differences that were evident at finer geographic levels. For example, only 72 per cent of Indigenous children in 'outer regional' areas of New South Wales were in excellent/very good health, whereas the same was true of 90 per cent of children in Queensland 'inner regional' areas.

\section{Hearing and vision}

Hearing impediments can delay speech and language development in children, with undesirable consequences for both social development and a child's ability to engage in educational opportunities. Previous studies have highlighted that hearing loss and impediments are more prevalent among Indigenous children (ABS 2006), particularly in more remote communities with poor environmental health conditions (Coates et al. 2002; Zubrick et al. 2004). Middle ear infection, or otitis media, is a persistent problem in many Indigenous communities and is regarded as the most common cause of hearing impediments among Indigenous children (Morris et al. 2005).

From the NATSISS, 9 per cent of children aged 0-14 years had an ear or hearing problem, which includes partial or full hearing loss and conditions such as tinnitus, runny/glue ear and tropical ear. The trend by age is roughly a reverse U-shape, peaking at age six (see Fig. 6.4). While we may have expected ear/ hearing problems to be more prevalent in remote areas, we found similar proportions in remote $(10 \%)$ and non-remote areas $(8 \%)$.

Only a small proportion (7\%) of children had an eye or sight problem. These problems were mainly of a less severe nature (long or short sightedness), with relatively few cases of blindness, trachoma, glaucoma, and cataracts. Similar to the findings of the Western Australian Aboriginal Child Health Survey, there were fewer cases of eye or sight problems in remote $(4 \%)$ than non-remote $(8 \%)$ areas (Zubrick et al. 2004). This is likely to reflect differences between remote and non-remote areas in the factors that are associated with short sightedness (for example, type of school work undertaken and lifestyle factors). 
Fig. 6.4 Proportion of Indigenous children with ear or hearing problems, by age, Australia, 2008a

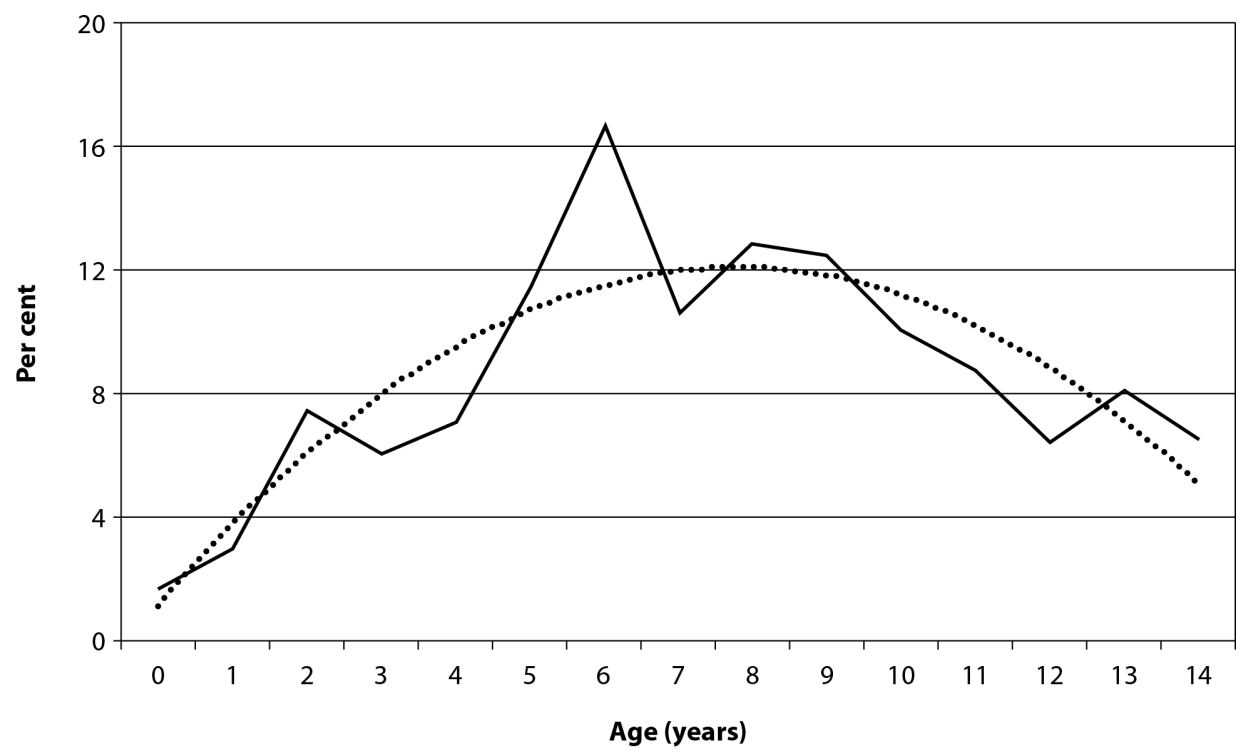

a. The broken lines represent a 'best fit' line, based on the coefficients of a second order polynomial regression.

Source: Authors' customised calculations using the 2008 NATSISS (accessed using the RADL)

Fig. 6.5 Proportion of Indigenous children with eye or sight problems, by age, Australia, 2008

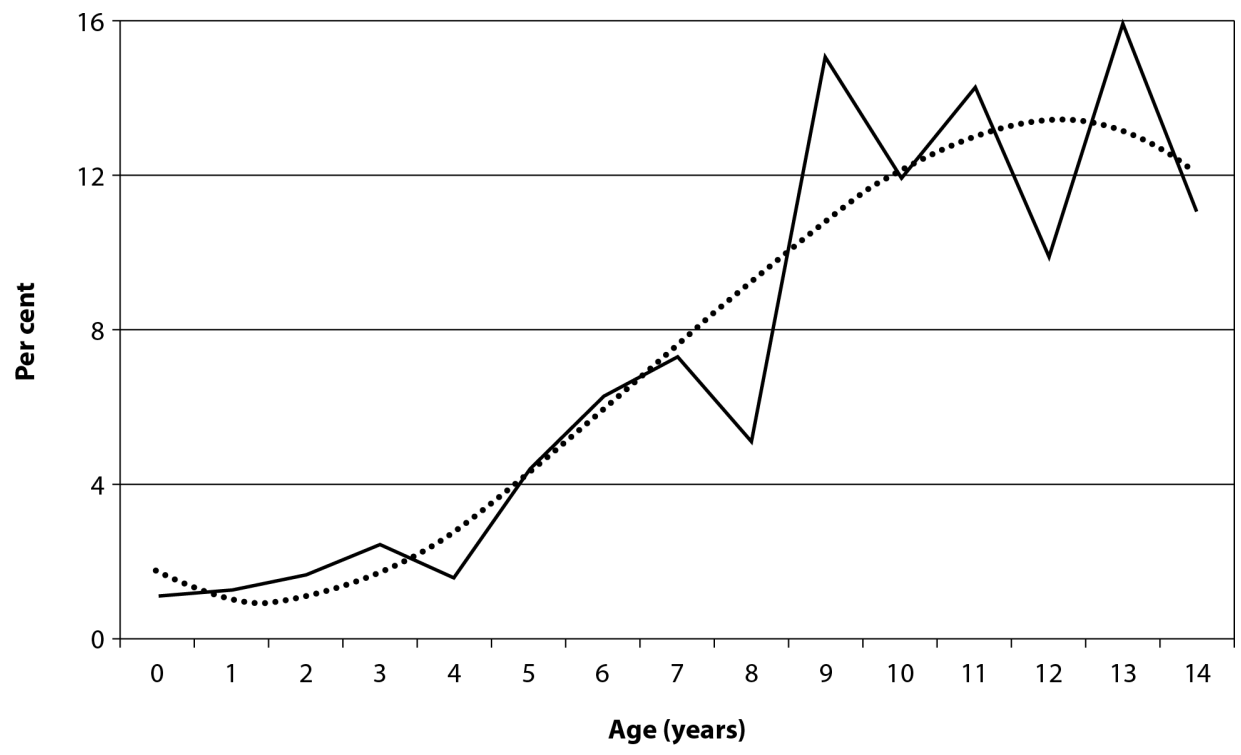

a. The broken lines represent a 'best fit' line, based on the coefficients of a third order polynomial regression. 


\section{Oral health}

About 36 per cent of children aged 2-14 years had at least one problem with their teeth or gums - this includes cavities, decay, fillings, breakage, having no teeth, and bleeding or sore gums. The NATSISS highlights that dental problems were less prevalent among children in remote settings (26\%) than non-remote areas (37\%), which may reflect a greater reliance on bush tucker in the most remote regions of Australia and a correspondingly smaller reliance on diets high in energy derived from refined carbohydrates and saturated fats (National Health and Medical Research Council 2000). This is consistent with the findings for Indigenous children in Western Australia (Zubrick et al. 2004) but contrasts the evidence of Jamieson Armfield and Roberts-Thomson (2007) in a study of Indigenous children in New South Wales, South Australia and the Northern Territory. The discrepancy between studies is likely to be attributable to differences in sample characteristics, collection methods, or the measurement of oral health between studies. The binary, carer-reported measure of dental problems used here may be a greater reflection of dental services use than dental problems per se. If so, then our findings would suggest that dental services are more accessible (and affordable) to Indigenous children living in less remote areas.

\section{Educational attendance}

The NATSISS design did not allow the collection of a robust range of variables on child education, and this naturally limits what can be examined in this important domain of child development. The survey questions mainly focus on issues of attendance, although the included items could only be considered proxy indicators of attendance patterns. Encouragingly, the vast majority of 'eligible' Indigenous children were going to school (97.5\%) and only a relatively small proportion of school children $(7 \%)$ were seen to have a problem with attendance (not attending without permission).

The carers of 27 per cent of school children stated that they had missed at least one day of school in the previous week, with the modal response for this group being five days (all days) missed. About 30 per cent of absence was due to sickness/injury, although many reported that the absence was because the school was not available or not open. These results are difficult to interpret but almost certainly support the observation that Indigenous students have poorer rates of attendance than their non-Indigenous counterparts (SCRGSP 2009).

Despite no data in the NATSISS on child academic performance, this outcome merits a short comment here. Other studies demonstrate clearly that there are considerable gaps in the performance of Indigenous and other children at school (SCRGSP 2009; Zubrick et al. 2006). Importantly, disparities are evident at Year 
1 and widen further in subsequent school years. These gaps are arguably the most important in terms of predicting onward disparities in human capabilities between Indigenous and other Australians.

Table 6.1 Proportion of Indigenous children with selected health/ development problems, Australia, 2008

\begin{tabular}{|c|c|c|c|}
\hline Health/development factor & $\begin{array}{c}\text { Non-remote } \\
(\%)\end{array}$ & $\begin{array}{l}\text { Remote } \\
\text { (\%) }\end{array}$ & $\begin{array}{c}\text { Total } \\
(\%)\end{array}$ \\
\hline \multicolumn{4}{|l|}{ Birthweight $^{a}$} \\
\hline Less than 2500 grams & 11.9 & 8.8 & 11.2 \\
\hline Less than 1500 grams & $2.1^{*}$ & $1.4 * *$ & $1.9 *$ \\
\hline Low gestation (less than 37 weeks) $^{a}$ & 23.8 & 25.2 & 24.1 \\
\hline \multicolumn{4}{|l|}{ Global health } \\
\hline Excellent & $48.0 \dagger$ & $41.4 \dagger$ & 46.4 \\
\hline Very good & $30.4 \dagger$ & $38.3 \dagger$ & 32.2 \\
\hline Good & 17.8 & 17.0 & 17.6 \\
\hline Fair & 3.0 & 2.6 & 2.9 \\
\hline Poor & $0.8 *$ & $0.8 *$ & 0.8 \\
\hline Eye or sight problem & $8.2 \dagger$ & $3.9+$ & 7.2 \\
\hline Ear or hearing problem & 8.0 & 10.2 & 8.5 \\
\hline Teeth or gum problems ${ }^{b}$ & $37.1 \dagger$ & $25.8 \dagger$ & 34.4 \\
\hline \multicolumn{4}{|l|}{ Educational attendance } \\
\hline 'Eligible' children not going to school' & 4.3 & 5.0 & 4.5 \\
\hline Problem with attendance ${ }^{d}$ & $5.4 \dagger$ & $12.8 \dagger$ & 7.0 \\
\hline
\end{tabular}
a. 0-3 year olds only.
b. 2-14 year olds only.
c. Excludes those who are too young, too old or ineligible for school.
d. Of those attending school.

$\dagger$ Denotes a statistically significant difference (at $95 \%$ level of confidence) in the proportions in remote and non-remote areas.

${ }^{*}$ Relative standard error between $25 \%$ and $50 \%$.

** Relative standard error greater than or equal to $50 \%$.

Source: Authors' customised calculations using the 2008 NATSISS (accessed using the RADL)

\section{Prompts, facilitators and constraints of child development}

As we outlined earlier, developmental strengths are influenced by a small set of mechanisms that either prompt, facilitate or constrain their development. 
- Developmental 'prompts' are particularly critical in the initiation of the acquisition and accumulation of skills. The developmental prompts of these skills include biology (including genes), expectations, and opportunities.

- Developmental 'facilitators' increase leverage from developmental prompts. These facilitators include: at least average intelligence; an easygoing temperament; emotional support in the face of challenge; and good language development.

- Developmental 'constraints' are those influences that impede or diminish the effects of the prompts or interact with the facilitators. These constraints include multiple accumulative stress, 'chaos' (i.e. war, social upheaval) that prevents the establishment of developmental stability, social inequality, and social exclusion.

Many of the factors that prompt or facilitate child development are either missing in the lives of Indigenous children or are too limited to produce sustainable benefits and opportunities in life. When skills and abilities are sufficiently acquired their benefits are, too often, constrained or overwhelmed by the influences of the living environment. Some of these constraints are characteristics of individuals or families, and have a direct influence on Indigenous children. Others are population-wide characteristics that impact on children in indirect ways, and reflect the fact that Indigenous populations have a diminished capability base relative to other Australians.

From a policy perspective, these prompts, facilitators and constraints offer avenues for deliberate investment at a variety of levels, from those that focus on individuals to those that affect national and global policy. There is plenty of flexibility to address them through one or more settings (e.g. family, school, care environments, work) using different instruments (legislation, remuneration, transfers and benefits, goods and services) to effect change.

\section{Population-wide constraints}

Population-wide constraints include lower life expectancy and higher fertility rates. These two factors conspire to produce a very young population (median age is 20 years) with a relatively low adult-to-child ratio. The NATSISS data highlight that there was 1.3 Indigenous adults (18+) for every Indigenous person aged 0-17 years, which compares with an approximate 3:1 ratio in the total population (ABS 2010). This indicates that Indigenous children have less access to older, experienced people available for care, protection, cultural guidance and general life-skills education (Silburn et al. 2006). This is compounded by high rates of imprisonment, father absence and family breakdown and consequent 
sole parent status. Over one-third (37\%) of Indigenous children less than one year of age were in one parent families; this proportion rose to 46 per cent among those aged 14 years.

Furthermore, the socioeconomic disadvantage experienced by Indigenous peoples in the form of low levels of education, employment and income, can generate stress. These circumstances change the capacity of populations to participate in, and benefit from, mainstream services (Zubrick et al. 2008). Indigenous children are vastly overrepresented in the lower levels of all socioeconomic constructs included in the NATSISS, including the area-based Socio-Economic Indexes for Areas (SEIFA) measure (see Fig. 6.6).

Fig. 6.6 Distribution of Indigenous children aged 0-14 years by SEIFA deciles, Australia, 2008

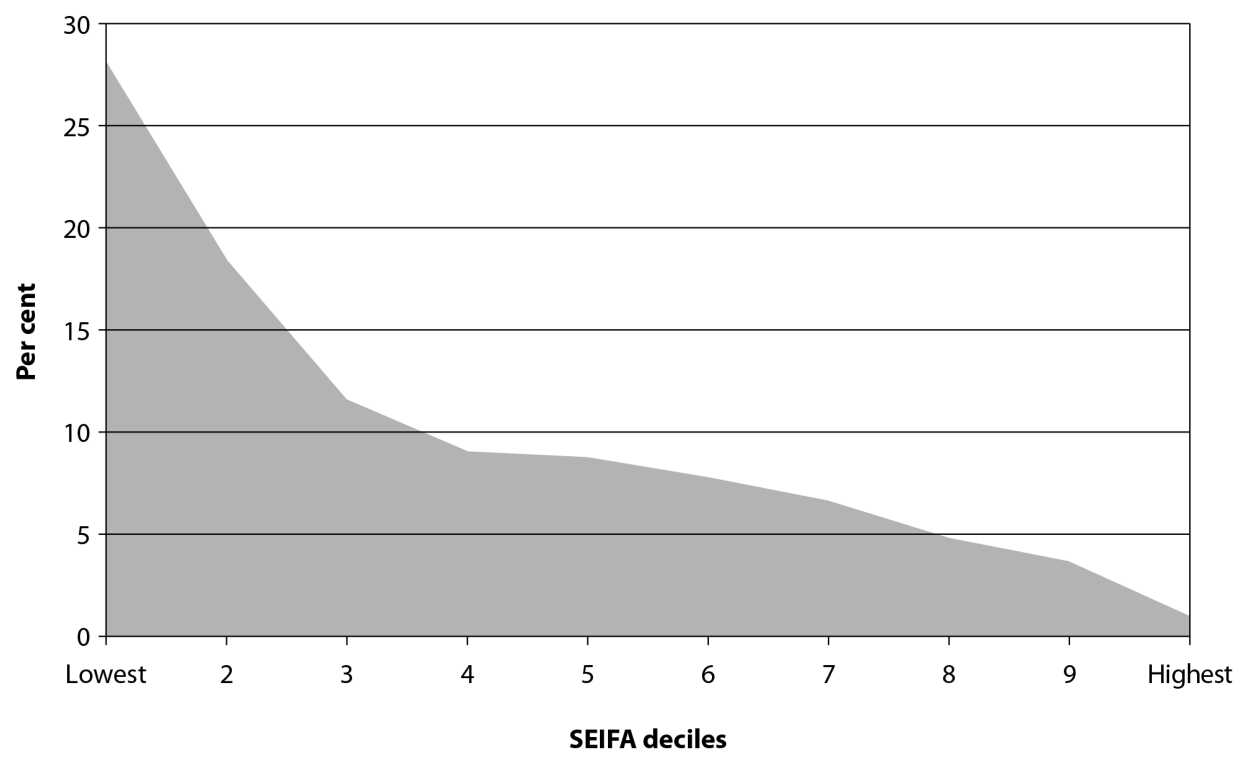

a. SEIFA deciles were determined based on the distribution of values for all Australian CDs. Source: Authors' customised calculations using the 2008 NATSISS (accessed using the RADL)

\section{A profile of prompts, facilitators and constraints}

Here we attempt to categorise 2008 NATSISS items as either prompts, facilitators or constraints of child development (see Table 6.2). The NATSISS cannot fully inform the breadth of these constructs nor are they necessarily the most salient measures. In some instances the selected items are proxy indicators of the constructs discussed earlier - for example, we use carer involvement 
in informal activities as an indicator of early language development. Despite these shortcomings, the NATSISS items, collectively, provide insight into the capability profile of Indigenous children in Australia.

The most prominent feature of the data presented in Table 6.2 is the high prevalence of development constraints. They document a profile of stress and discrimination that are experienced at levels unique to Indigenous children. For example, 44 per cent of 0-3 year olds and 65 per cent of 4-14 year olds experienced at least one of the stressors that were asked about in the NATSISS. These stressors commonly included serious events such as the death of a close family member/friend, having a really bad illness/accident, and being physically hurt by someone. When these types of stressors occur frequently in early life they can have serious longer-term effects on the development of the brain, endocrine and immune systems, and are a key mechanism in the biological embedding of disadvantage (McEwen 1998). Carers also reported that 15 per cent of school children aged 6-14 years were bullied or treated unfairly at school because they were Indigenous, 9 per cent needed to stayed overnight somewhere else due to a family crisis in the six months prior to the survey, and 62 per cent of 5-14 year olds had moved house in the last five years.

Table 6.2 Summary of selected developmental prompts, facilitators and constraints of Indigenous child development, by remoteness, Australia, 2008

\begin{tabular}{|c|c|c|c|}
\hline & $\begin{array}{c}\text { Non-remote } \\
(\%)\end{array}$ & Remote (\%) & Total $(\%)$ \\
\hline \multicolumn{4}{|c|}{ Developmental prompts } \\
\hline \multicolumn{4}{|l|}{ Birthweight $^{\mathrm{a}}$} \\
\hline Less than 2,500 grams & 11.9 & 8.8 & 11.2 \\
\hline Less than 1,500 grams & $2.1^{*}$ & $1.4 * *$ & $1.9 *$ \\
\hline \multicolumn{4}{|l|}{ Breastfeeding $^{a}$} \\
\hline Never been breastfed & $24.6 \dagger$ & $13.7 \dagger$ & 22.2 \\
\hline Breastfed but less than 3 months & $23.2 \dagger$ & $10.0 \dagger$ & 20.3 \\
\hline Does not usually eat fruit ${ }^{b}$ & $4.8 \dagger$ & $2.3+$ & 4.2 \\
\hline Does not usually eat vegetables ${ }^{b}$ & 3.5 & 2.4 & 3.3 \\
\hline $\begin{array}{l}\text { Identified with a clan, tribe or language } \\
\text { group }^{c}\end{array}$ & $40.6 \dagger$ & $69.2 \dagger$ & 47.4 \\
\hline $\begin{array}{l}\text { Some involvement in cultural events, } \\
\text { ceremonies or organisations in last } 12 \\
\text { months }^{c}\end{array}$ & $66.5 \dagger$ & $80.9 \dagger$ & 70.0 \\
\hline Participation in cultural activities ${ }^{c}$ & $60.4 \dagger$ & $79.4 \dagger$ & 64.9 \\
\hline \multicolumn{4}{|c|}{ Developmental facilitators } \\
\hline \multicolumn{4}{|l|}{ Education of main carer } \\
\hline Completed Year 12 & 22.8 & 19.4 & 22.0 \\
\hline Non-school qualification & $38.2 \dagger$ & $23.6 \dagger$ & 34.8 \\
\hline
\end{tabular}




\begin{tabular}{|l|c|c|c|}
\hline \multicolumn{5}{|c|}{$\begin{array}{c}\text { Non-remote } \\
\text { (\%) }\end{array}$} \\
\hline Time spent by main carer doing informal learning activities with child in last week \\
\hline None & 3.3 & $4.1^{\text {d }}$ & 3.5 \\
\hline $1-6$ days & 26.3 & 26.5 & 26.3 \\
\hline 7 days & 70.2 & 69.1 & 69.9 \\
\hline Type of informal learning activities main carer did with child in last week \\
\hline Read a book (0-6 year olds) & $74.7 \dagger$ & $54.6 \dagger$ & 69.8 \\
\hline Told a story (0-6 year olds) & 60.1 & 60.7 & 60.3 \\
\hline Listened to child read (7-10 year olds) & $71.6 \dagger$ & $53.3 \dagger$ & 67.2 \\
\hline \multicolumn{4}{|c|}{ Developmental constraints } \\
\hline Experienced a stressor in last 12 months & $46.6 \dagger$ & $35.1 \dagger$ & 44.0 \\
\hline 0-3 year olds & 66.3 & 59.8 & 64.8 \\
\hline 4-14 year olds & $16.1 \dagger$ & $10.8 \dagger$ & 14.9 \\
\hline Bullied or treated unfairly at school & & \\
\hline $\begin{array}{l}\text { Stayed overnight somewhere else due to } \\
\text { family crisis in the last 6 months }\end{array}$ & 9.1 & 9.6 & 9.2 \\
\hline $\begin{array}{l}\text { Affected by friends/family members with } \\
\text { alcohol problem }\end{array}$ & 11.6 & 13.2 & 12.0 \\
\hline $\begin{array}{l}\text { Affected by friends/family members with } \\
\text { drug problem }\end{array}$ & 9.1 & 8.2 & 8.9 \\
\hline Moved house in the last 5 years & & 62.0 \\
\hline Needed more formal child care ${ }^{9}$ & 63.3 & 57.6 & 13.8 \\
\hline
\end{tabular}
a. 0-3 year olds.
b. 1-14 year olds.
c. 3-14 year olds.
d. 1-6 year olds.
e. 2-14 year olds that were attending school.
f. 5-14 year olds.
g. 0-12 year olds.

$\dagger$ Denotes a statistically significant difference (at $95 \%$ level of confidence) in the proportions in remote and non-remote areas.

${ }^{*}$ Relative standard error between $25 \%$ and $50 \%$.

${ }^{* *}$ Relative standard error greater than or equal to $50 \%$.

Source: Authors' customised calculations using the 2008 NATSISS (accessed using the RADL) 


\section{Associations with child developmental outcomes: An example using socioeconomic constructs}

To this point we have presented some indicators of Indigenous child development, described the mechanisms that prompt, facilitate and constrain development and outlined some general principles for improving human capabilities in Indigenous contexts. Here we provide an insight into the relative importance of factors that influence Indigenous child development.

There is a relatively circumscribed literature on the nature of the associations between Indigenous child developmental outcomes and their antecedents. The empirical evidence suggests that there is a fairly weak relationship between income, education and employment of Indigenous adults and the developmental outcomes of their children (Zubrick et al. 2005). We test this observation with 2008 NATSISS data and focus on three constructs of socioeconomic status and their association with the overall (global) health of children: the educational attainment of the main carer of the child, household income (equivalised), and area-level relative disadvantage (SEIFA). We chose these for this analysis as they represent three different dimensions of socioeconomic status at multiple levels (parent, family and neighbourhood). The result of greatest interest is children who are reported to have excellent or very good health at the time of the survey. The shape and magnitude of the associations between socioeconomic status and child health is highlighted by Fig. 6.7, which shows the odds ratios from logistic regression analyses. There appears to be no association between the parent-rated measure of child health status and carer education and no statistically significant trend by the SEIFA measure, although those children in the third quintile of SEIFA were 1.4 times more likely (95\% CI: 1.31-1.46) to be in excellent or very good health than those in the lowest quintile. Household income is positively associated with child health, although the relationship is non-linear in nature and only features an elevated odds of having excellent or very good health for children in the top two quintiles (OR $=1.8 ; 95 \% \mathrm{CI}: 1.64-1.95)$ and second quintile (OR $=1.5 ; 95 \%$ CI: 1.39-1.54), relative to those in the lowest quintile. Household income continues to have a statistically significant independent effect on child health when analysed collectively with carer education and SEIFA $(\mathrm{p}=0.0024)$. 
Fig. 6.7 Relative odds of excellent or very good health in Indigenous children, by constructs of socioeconomic status, Australia, 2008 ${ }^{a}$

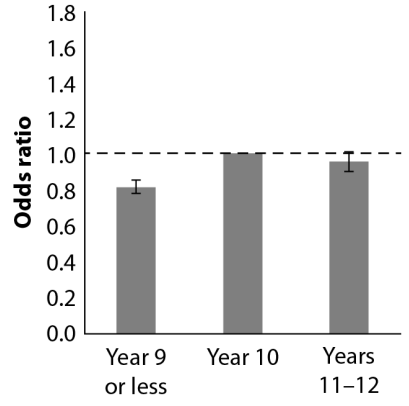

Carer education
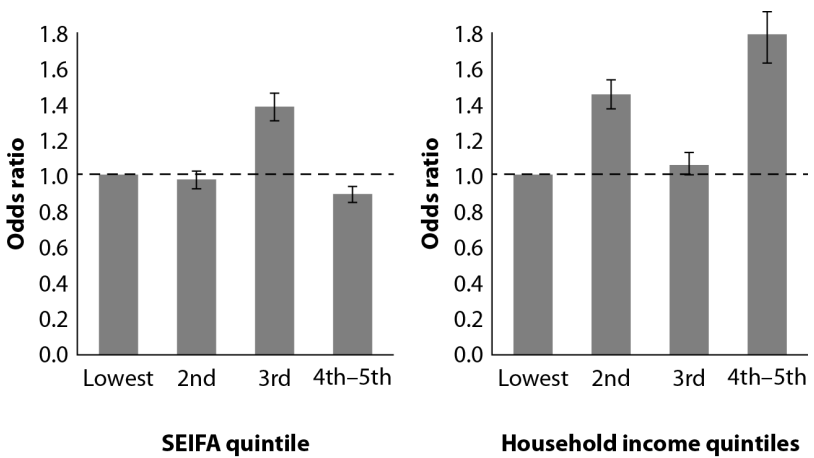

a. All logistic regression models include age and sex as covariates. Household income is derived using equivalence scales; quintiles have been derived based on the distribution of total household income for Indigenous and non-Indigenous households. SEIFA quintiles were determined based on the distribution of values for all Australian CDs.

Source: Authors' customised calculations using the 2008 NATSISS (accessed using the RADL)

Fig. 6.8 Relative odds of excellent or very good health in Indigenous children, by household income, Australia, 2008: Simple and full modelsa

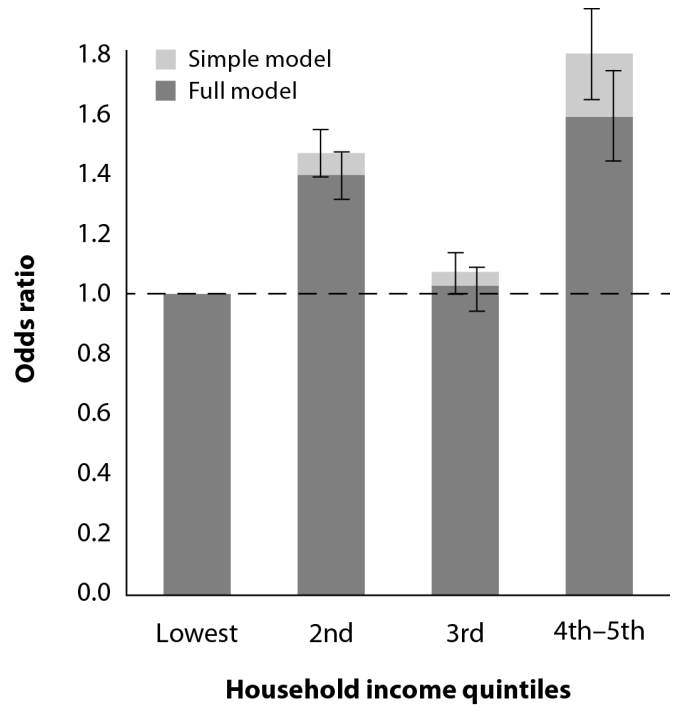

a. 'Simple' logistic regression model includes age and sex as covariates. The 'full' model also includes the following covariates: remoteness, SEIFA quintiles, carer education, experience of stressors, carer engagement in informal activities with the child, whether bullied or treated unfairly, whether child stayed overnight somewhere else because of family crises, whether child was involved in cultural events, ceremonies or organisations or participated in cultural activities in last 12 months, and whether child eats fruit and vegetables. Household income is derived using equivalence scales; quintiles have been derived based on the distribution of total household income for Indigenous and non-Indigenous households.

Source: Authors' customised calculations using the 2008 NATSISS (accessed using the RADL) 
The association between household income and child health is only slightly attenuated by the inclusion of other factors in the model that are known to influence health in early life (see Fig. 6.8). We found that stress, carer engagement in informal activities with the child, and bullying and discrimination were all factors significantly associated with a child being in excellent or very good health, and that household income has an effect on child health over and above the influence of these factors.

\section{Implications}

Our findings suggest that the strong associations characteristically seen in mainstream populations between child health and development outcomes and socioeconomic status do not necessarily hold in Indigenous populations. This does not imply that these factors are unrelated to the development of Indigenous children or that improving education, for example, is unwarranted. Instead it is likely to reflect that there are other circumstances in the social and physical environment that disrupt these associations for large segments of the Indigenous population (Zubrick et al. 2008).

Weak health gradients are particularly problematic for populations with low levels of health because they imply that there are greater barriers to improving health. If traditional levers do not produce improvements in health then these populations are in danger of being 'trapped' in poor health (Buttenheim et al. 2010). It is difficult to underestimate the implications of this for Indigenous Australia. The current policy imperative is one that aims to 'close the (developmental) gap' between the mainstream and Indigenous population within a generation (Department of Families, Housing, Community Services and Indigenous Affairs 2009). These findings of weak associations between determinants of human development and human capital formation would suggest that either the policy expectation is overly ambitious or that greater effort will be needed to compensate for the reduced effect size.

The findings here confront policy and practice settings with competing demands: the urgency to be seen to be 'doing something' to address the acute needs and demands of families overwhelmed by crises while at the same time diverting government resources and energies to the longer and slower process of enabling demographic restitution of capability. As noted above, this process is commencing from a very low base and it is unlikely that there is any generational short-cut in the time that it will take to effect true change. We have highlighted that Indigenous children have less access to older, experienced people available for their care, protection, cultural guidance and general life-skills education. The 'treatment' for this is primarily a demographic treatment: delay the onset of age of first pregnancy while concurrently increasing the proportion of Indigenous children that receive high quality early childhood educational daycare and 
support into primary school. The goal here is to prolong enrolment, attendance and retention into the upper secondary school to increase the proportion of the Indigenous population that has vocational and tertiary experiences - this will build greater human capital. It will have the ultimate effect of expanding choices for Indigenous adults and, concomitantly, improve the wellbeing and life opportunities of Indigenous populations.

While this is slowly transforming the capability profile of the Indigenous population, there is a need to specifically enrol Indigenous people in understanding how to reduce the developmental chaos which is the major constraint affecting Indigenous children. This will take different forms depending on where the child and family are living - the major areas here are demarked by the metropolitan setting (urban), transition zone (rural and remote regional centres) and extremely remote areas. The short-term strategies require establishing effective buffering around the child and stabilising the level of chaos the child is exposed to: reducing the effects of direct and indirect violence, improving the quality of the material environment particularly for children aged 2-4 years, establishing emotional support for the adult carer, and providing regularity in routine and setting realistic expectations for the child. The treatment for the population is a focus on slow, progressive, upstream and distal changes in human capital formation; the treatment for children living today is a proximal approach with an explicit engagement of Indigenous adults in enhancing life prospects.

\section{Conclusion}

We have been able to utilise the 2008 NATSISS to explore the developmental status of Indigenous children in Australia. We have demonstrated three significant results from the 2008 NATSISS data. First, the majority of Indigenous children are in excellent or very good overall health, although there are some developmental danger signs - that are evident from birth - for a significant number of children. Second, the profile of developmental constraints in Indigenous Australia is likely to overwhelm the critical acquisition of skills and abilities for many children. This analysis confirms that stress and discrimination are part of many Indigenous children's lives, and from an early age. Third, the associations between child development outcomes and determinants of human development may be weaker in Indigenous populations relative to mainstream Australia. This suggests that policy responses that are suitable for the general population need to be modified in order to significantly benefit the health of Indigenous peoples. 
There are a number of limitations to what we have presented here. The crosssectional nature of the NATSISS, the limited information on aspects of education, social and emotional wellbeing and geography, and the use of questions that rely on carer perception and recall, has curbed the breadth of potential analyses and what can be inferred from our results.

\section{Acknowledgement}

Carrington Shepherd is financially supported by a Sidney Myer Health Scholarship.

\section{References}

Australian Bureau of Statistics (ABS) 2006. National Aboriginal and Torres Strait Islander Health Survey, 2004-05, cat. no. 4715.0, ABS, Canberra.

2010. Population by Age and Sex, Australian States and Territories, Jun 2010, cat. no. 3201.0, ABS, Canberra.

Australian Institute of Health and Welfare (AIHW), Mathers, C., Vos, T. and Stevenson, C. E. 1999. The Burden of Disease and Injury In Australia, cat. no. PHE 17, AIHW, Canberra.

Bell, S. 1995. 'Building Aboriginal health from the ground upwards', Paper presented at the Aboriginal Health: Social and Cultural Transitions Conference, viewed 7 May 2010, available at $<$ www.caac.org.au/pr/index.php?dl=28>

Boddington, P. and Raisanen, U. 2009. 'Theoretical and practical issues in the definition of health: Insights from Aboriginal Australia', Journal of Medicine and Philosophy, 34 (1): 49-67.

Briscoe, G. 2003. Counting, Health and Identity: A History of Aboriginal Health and Demography in Western Australia and Queensland, Aboriginal Studies Press, Canberra.

Bronfenbrenner, U. 1979. The Ecology of Human Development: Experiments by Nature and Design, Harvard University Press, Cambridge, Massachusetts.

Buttenheim, A., Goldman, N., Pebley, A. R., Wong, R. and Chung, C. 2010. 'Do Mexican immigrants "import" social gradients in health to the US?', Social Science and Medicine, 71 (7): 1268-76. 
Coates, H., Morris, P., Leach, A. and Couzos, S. 2002. 'Otitis media in Aboriginal children: Tackling a major health problem', Medical Journal of Australia, 177 (4): 177-78.

Department of Families, Housing, Community Services and Indigenous Affairs 2009. Closing the Gap on Indigenous Disadvantage: The Challenge for Australia, Australian Government, Canberra.

Ford, J., Nassar, N., Sullivan, E. A., Chambers, G. and Lancaster, P. 2003. Reproductive Health Indicators, Australia 2002, cat. no. PER 20, AIHW National Perinatal Statistics Unit, Canberra.

Fukuda-Parr, S. and Kumar, A. K. S. 2004. Readings in Human Development: Concepts, Measures and Policies for a Development Paradigm, Second ed., Oxford University Press, New Delhi.

Jamieson, L. M., Armfield, J. M. and Roberts-Thomson, K. F. 2007. 'Indigenous and non-indigenous child oral health in three Australian states and territories', Ethnicity and Health, 12 (1): 89-107.

Jessor, R. 1993. 'Successful adolescent development among youth in high-risk settings', American Psychologist, 48 (2): 117-26.

Keating, D. P. and Hertzman, C. 1999. Developmental Health and the Wealth of Nations: Social, Biological, and Educational Dynamics, Guilford Press, New York.

Kuh, D. and Ben-Shlomo, Y. 2004. A Life Course Approach to Chronic Disease Epidemiology, Second edn, Oxford University Press, New York.

McEwen, B. S. 1998. 'Protective and damaging effects of stress mediators', New England Journal of Medicine, 338 (3): 171-79.

Morris, P., Leach, A., Silberberg, P., Mellon, G., Wilson, C., Hamilton, E., et al. 2005. 'Otitis media in young Aboriginal children from remote communities in Northern and Central Australia: A cross-sectional survey', BMC pediatrics, 5 (1): 27 .

National Health and Medical Research Council 2000. Nutrition in Aboriginal and Torres Strait Islander Peoples: An Information Paper, National Health and Medical Research Council, Canberra.

Saggers, S. and Gray, D. 2007. 'Defining what we mean', in B. Carson, T. Dunbar, R. D. Chenhall and R. Bailie (eds), Social Determinants of Indigenous Health, Allen \& Unwin, Sydney.

Sen, A. 2002. 'Health: Perception versus observation', BMJ, 324 (7342): 860-61. 
Silburn, S. R., Zubrick, S. R., De Maio, J. A., Shepherd, C., Griffin, J. A., Mitrou, F. G., et al. 2006. The Western Australian Aboriginal Child Health Survey: Strengthening the Capacity of Aboriginal Children, Families and Communities, Telethon Institute for Child Health Research, Perth.

Steering Committee for the Review of Government Service Provision (SCRGSP) 2009. Overcoming Indigenous Disadvantage: Key Indicators 2009, Productivity Commission, Canberra.

Young, M. E. 2002. Early Child Development:Investing in the Future, World Bank, Washington DC, viewed 29 September 2011, <http://siteresources.worldbank. org/EDUCATION/Resources/278200-1099079877269/547664-1099079922573/ ECD_investing_in_the_future.pdf $>$

Zubrick, S. R. 2010. 'Where do strengths come from? The contribution of childhood in the course of human development', Paper presented at the 6th National Family and Community Strengths Conference, NovemberDecember 2010, University of Newcastle, Newcastle.

- Lawrence, D. M., Silburn, S. R., Blair, E., Milroy, H., Wilkes, T., et al. 2004. Western Australian Aboriginal Child Health Survey: The Health of Aboriginal Children and Young People, Telethon Institute for Child Health Research, Perth.

—, Silburn, S. R., De Maio, J. A., Shepherd, C., Griffin, J. A., Dalby, R. B., et al. 2006. The Western Australian Aboriginal Child Health Survey: Improving the Educational Experiences of Aboriginal Children and Young People, Telethon Institute for Child Health Research, Perth.

—, Silburn, S. R., De Maio, J., Shepherd, C., Griffin, J. A., Dalby, R. B., et al. 2008. 'The Western Australian Aboriginal Child Health Survey: Are there any policy implications?', in G. Robinson, U. Eickelkamp, J. Goodnow and I. Katz (eds), Contexts of Child Development - Culture, Policy and Intervention., Charles Darwin University, Darwin.

—_ Silburn, S. R., Lawrence, D. M., Mitrou, F. G., Dalby, R. B., Blair, E. M., et al. 2005. The Western Australian Aboriginal Child Health Survey: The Social and Emotional Wellbeing of Aboriginal Children and Young People, Telethon Institute for Child Health Research, Perth.

, Taylor, C. L., Lawrence, D., Mitrou, F., Christensen, D. and Dalby, R. 2009. 'The development of human capability across the lifecourse: Perspectives from childhood', Australasian Epidemiologist, 16 (3): 6-10. 